\title{
Research on Diffluent Network Prediction Model of Ecotourim Scenic Spot Based on the Dynamic Fuzzy Control
}

\author{
Peng Ge, Peiyu Ren*, Maozhu Jin, Yanqing Qiu and Weimin Zheng \\ Business School of Sichuan University, Chengdu 610065, China
}

Received: 5 Mar. 2013, Revised: 8 Jul. 2013, Accepted: 9 Jul. 2013

Published online: 1 Nov. 2013

\begin{abstract}
The acute contradiction between tourism economic development and ecological environment protection is becoming increasingly serious in many natural scenic spots. It became the focus of both managers of scenic area and scholars that using advanced information technology to track the swath law of tourists and realizing real-time control of the time and space distribution of visitors in scenic area. This paper adopted the idea of fuzzy control, constructed a dynamic fuzzy feedback control forecast model based on the adaptive laws at first, then it proved two kinds of effects existed in this model. Finally, it simulated a single units attraction, and the result of comparative analysis with current state of experience based schedule proved the effectiveness of the system proposed in this paper. This formed the foundation for the real-time scheduling in future study.
\end{abstract}

Keywords: Ecotourism, Fuzzy Control, Dynamic Diffluent Network, Traffic Congestion, Environment Protection.

\section{Introduction}

For Natural Scenic Areas, fully enjoy the natural scenery is the key factor that effects the quality of the tourist experience. And it is tourism development tendency that experience tourism will replace normal tourism, so the visitors experience quality plays a crucial role to the sustainable development of the scene. The Week-long Statutory Holidays of China leads to the travel peak of scenic area. This phenomenon often leads to the overload of local areas transient in addition to the poor management on the tourists distribution in scenic area. These overloads broke the ecological environment of the local areas irreversibly, and brought about the un-satisfaction of tourists. Its only the local behavior if we do the vehicle schedule by tourists personal intention, and this kind of behavior will enhance the chaos of the system, increase the system entropy, and finally create management confusion of the system [1]. It become a focus of attention that how to balance the contradiction between the government and the scenic area managers.

Scenic spot shunt network has the features of complicated system: 1) large quantity of detection information, involving a lot of variables [2]; 2) the cohesion relation among the passenger flows [3]; 3) the uncertainty of the information [1]; 4) system has the chaotic attribute: butterfly effect, and any tiny decision deviation of a single spot are likely to cause serious deviation of the system demand, then mislead the whole schedule effect. In addition, the quantity of visitors in every attraction changes at any time, and tourists travel activity assumes to be like the Herd Behavior. All these will make the system state out of our expectation. It is necessary to do some feedback control with dynamic prediction, and take real-time schedule steps to ensure the stability of the system. Scenic spot scheduling problem is actually a traffic flow problem with lots of nodes. There has already existing research confirmed that traffic flow are obviously chaos phenomena [4]. Jiyuan Peng et al. (2005) [5] took the operation scheme of local circulation, and established the vehicle scheduling scheme for scenic spot at fastigium according to the historical experience. That was a static plan and it didnt take the real-time influence of tourist on attractions state into consideration. We plan to analyze the attractions characteristics of space and time in a tourist cycle. Then realize the load balance of attractions with the artificial schedule. It is well known that, the control of the movement started from the

\footnotetext{
* Corresponding author e-mail: gepeng @ scu.edu.cn
} 
anticipation of expected output motion of a system [6]. Therefore, it is necessary to master the real time state of the whole system, and establishing the real-time prediction control engine is a good way. Predictive control is a control algorithm based on the prediction model. It can predict the future output of a system according to its current input and its history information [7]. There are many ways to deal with control problem of chaos system, such as delay feedback control, adaptive control, neural network, fuzzy control, etc., but most of these methods are for physical system [8-10]. The uncertainty of the passenger flow volume between any two attractions is more and more aggravate as the scale of tourists grow up. It is difficult to trace real- time change of passenger flow accurately for simple time series prediction. Many causal prediction algorithms with no model (consider other factors such as passenger flow relationship between attractions) have been developed in recent years. Actually, there will be more updated forecast technology which can be applied to the flow prediction problems in short time domain along with the development of intelligent control and guidance system [11]. Mingbao Pang et al. (2009) [12] put forward a highway chaotic control method which was the combination of delay feedback and fuzzy control. They also verified the effectiveness of the method through simulation experiments. With this idea, we use the fuzzy concept crowded degree to describe the state of attractions at any given instant. We also establish the fuzzy control model of single attraction based on the adaptive law, and try to realize the real-time control of the whole system by importing external law and lumped law. Finally, we use MATLAB to simulate the fuzzy control model, forecast the dynamic behavior and real-time capacity demand of the system, analyze the system performance of controller. All these are used to support the diversion scheduling strategy of scenic spot.

\section{Dynamic fuzzy feedback forecast model}

We divide the fuzzy control network into three levels according to the complex characteristics of our system. Take Figure1 for example, (1) every attraction $\left(A_{1}-A_{3}\right.$, $\left.B_{1}-B_{4}\right)$ is not only a proposer of control demand, but also the controlled object, we call them the fuzzy control unit (for short, unit); (2) passenger flow is formed by taking schedule actions to the control units, then part of (see line with arrows $b_{23} \& b_{24}$ in Figure 1) or all (see line with arrows $a_{12}, a_{23} \& b_{12}$ in Figure1) the output of upstream unit form the input of its downstream unit. Then the feedback control of a single unit will affect the state of its upstream unit, many such control units interact to form Multi-control agency (see A \& B in Figure1); (3) from the entire scenic point of view, we should balance the loads of attractions in order to maximize the scale of tourists within the limit of its capacity. Therefore, we need to monitor the equilibrium of system in real time, and call

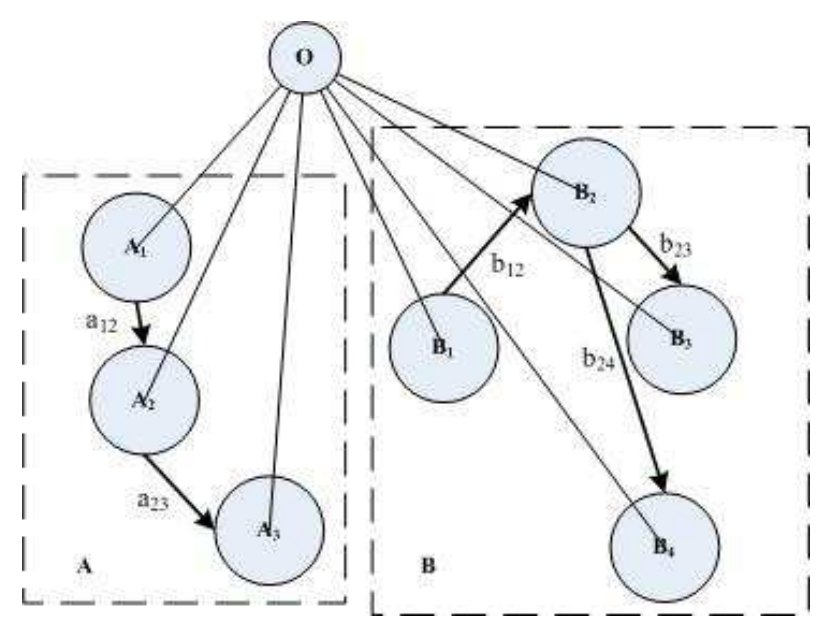

Fig. 1: Hierarchical construction of fuzzy control network

this lumped control (that is, every unit of the whole system is adjusted by node $\mathrm{O}$ ).

\section{Fuzzy control unit}

T. Takagi et al. (1985) [13] first introduced the fuzzy recognition into system control. The congestion degree of attractions is the main fuzziness of our object. According to the capacity calculation method of Xiaoping Zhang et al. (2007) [14], we can calculate the instant capacity of attraction is static (the instant capacity of attraction may vary with the major changes of ecological environment, but it will not effect in our study). Define the load of attraction is the ratio of its tourists and capacity at a time, though the load fluctuates with tourists, it is a certain value, while the crowded degree is a fuzzy concept. In addition, for a single person, the time to visit a certain attraction is fixed, but for a tourist group, it is not only about the individual differences, but also the crowded degree of the attraction. Its an embedded random variable, and is out of the scope of this paper.

Figure 2 shows a simple fuzzy control unit. Through the adaptive law, the crowded degree feedbacks to input and form the crowded state of system with shunt rate together. It will slow down the convergence rate and is useless for system control if the adaptive law is too small, and will damage the stability of system otherwise. G. J. Kulawski et al. (2000) [15] first gave the setting solution of adaptive law for uncertain nonlinear system through the dynamic neural network. We use adaptive law, external law and lumped law to represent the feedbacks, and do assignment separately according to the simulation of real data.

Set $C_{i}$ be the instant capacity for attraction $i, x_{i}^{t}$ be the tourists in attraction $i$ at instant $t$, then the load of attraction $i$ at instant $t$ can be expressed as $R_{i}^{t}=x_{i}^{t} / C_{i}$. Assume $\lambda_{i}^{t}$ be the arrival rate of tourists in attraction $i$ at instant $t, \mu_{i}^{t}$ be 


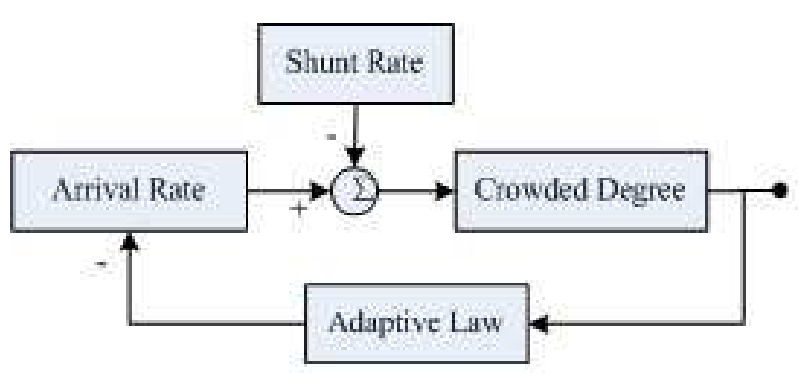

Fig. 2: Flow chart for fuzzy control unite

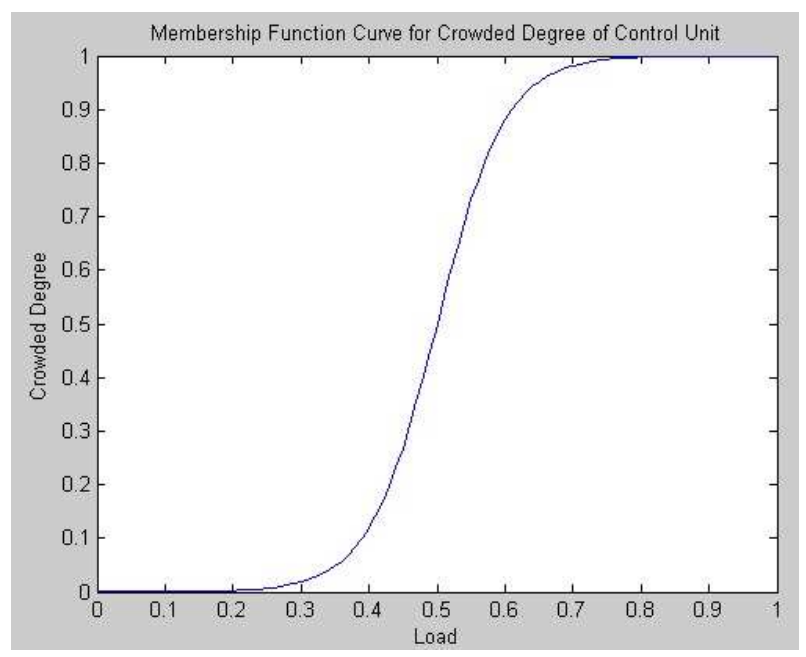

Fig. 3: Membership function for crowded degree of unit

its shunt rate, then the increment of tourists in attraction $i$ is $x_{i}^{t}=\lambda_{i}^{t}-\mu_{i}^{t}$. Other parameters are defined below:

Definition 1 Crowded Degree $c r d$ : Obfuscation of the load of attractions, reflecting the busy state of attractions at a certain time. ExpectedCrowdedDegree is expressed by $\mathrm{crd}^{*}$, and its usually a known number. Function Sigmf can describe a complete fuzzy concept, such as high or low of water level, fat or thin of people etc. [16]. According to the subjective experience method, we use Function Sigmf (as shown in Figure 3) to describe the Crowded Degree of attraction $i$ at instant $t$ : $c r d_{i}^{t}=\frac{1}{1+e^{-2\left(R_{i}^{t}-5\right)}}$. We give the fuzzy control rule here: IF $c r d_{i}^{t} \leq c r d^{*}$, THEN reduce $\lambda_{i}^{t}$, ELSE increase $\lambda_{i}^{t}$. And the amount of variation is controlled by adaptive law.

Definition 2 Adaptive Law par: The adjustment function for the crowded degree of control unit acts on its arrival rate. It changes according to the deviation from the state to its expectations generally.

\subsection{Multi-control agency}

The crowded degree of fuzzy control unit can not only control its own arrival rate through the adaptive law, but

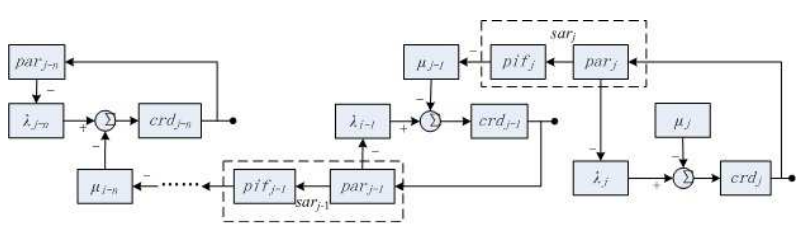

Fig. 4: Flow chart of domino effect of n-control agency

also can control the shunt rate of its upstream unit. Multi-control Agency is formed of many control units by the roles of these laws. In real system, passenger flows between attractions interact with each other, and this can be simplified as a distributed feedback control system with serial and parallel relations. Set pif be the shunt probability of a certain attraction, then the arrival rate of the target control unit $=$ the shunt rate of its upstream unit * pif. We focuses on serial feedback control system in this paper.

Definition 3 External Law sar: In Multi-Control Agency, part of the shunt rate of the upstream unit may make up part of the arrival rate of the downstream unit. Therefore, the regulation of adaptive law on its arrival rate can also regulate the shunt rate of its upstream unit with the effect of shunt probability.

Property 1 Domino Effect: External Law has the characteristic of reverse transfer, i.e., the variation of the crowded degree of a single control unit will modify the shunt rate of its upstream unit, and the regulation is delayed.

Proof: Given a Multi-Control Agency with $n$ fuzzy control unit and the original value of parameters are shown in Figure 4. Prove that the crowded variation of control unit $j$ at instant $t\left(\Delta c r d_{j}^{t}\right)$ will modify the shunt rate of its upstream unit $j-1$ at instant $t+1\left(\mu_{j-1}^{t}\right)$ through the external law $\left(s a r_{j}\right)$. Deduce the recursive formula of the correct factors in an $n$-Control Agency.

From Figure 4, we can conclude that sar $=$ par $\cdot$ pif. Set the crowded degree error of control unit $j$ at instant $t$ : $\varepsilon_{j}^{t}=c r d_{j}^{t}-c r d^{*}$, add variation $\Delta c r d_{j}^{t}$ to it, then $\varepsilon_{j}^{t+1}=\varepsilon_{j}^{t}+$ $\Delta c r d_{j}^{t}$, according to the Feedback Control Principle [17], the correction of $\Delta c r d_{j}^{t}$ for the shunt rate of control unit $j-1$ at instant $t\left(\mu_{j-1}^{t}\right)$ can be expressed as $r_{j}^{t+1}=\operatorname{par}_{j}$. $\operatorname{pif}_{j} \cdot\left(\operatorname{crd}_{j}^{t+1}-\operatorname{crd}^{*}\right)=\operatorname{sar}_{j} \cdot \varepsilon_{j}^{t+1}$, then $\mu_{j-1}^{t+1}=\mu_{j-1}^{t}-r_{j}^{t}=$ $\mu_{j-1}^{t}-\operatorname{sar}_{j} \cdot \varepsilon_{j}^{t+1}=\mu_{j-1}^{t}-\operatorname{sar}_{j} \cdot\left(\varepsilon_{j}^{t}+\Delta c r d_{j}^{t}\right)$, we can see that $\Delta c r d_{j}^{t}$ modified $\mu_{j-1}^{t}$ at $t+1$.

And the like, the shunt rate of the $n+1$ control unit before $j$ is $\mu_{j-n-1}^{t+n+1}=\mu_{j-n-1}^{t+n}-r_{j-n}^{t+n}$, and its correct factor is $r_{j-n}^{t+n}=\operatorname{sar}_{j-n} \cdot \varepsilon_{j-n}^{t+n}=\operatorname{sar}_{j-n} \cdot\left(\varepsilon_{j-n}^{t+n-1}+\Delta c r d_{j-n}^{t+n-1}\right)$,

$$
\begin{aligned}
& \Delta c r d_{j-n}^{t+n-1} \\
& =\frac{1}{1+e^{-2\left(\left(\lambda_{j-n}^{t+n-1}-\mu_{j-n}^{t+n-1}+x_{j-n}^{t+n-1}\right) / C-5\right)}}-c r d_{j-n}^{t+n-2} \\
& =c r d_{j-n}^{t+n-1}-c r d_{j-n}^{t+n-2}
\end{aligned}
$$




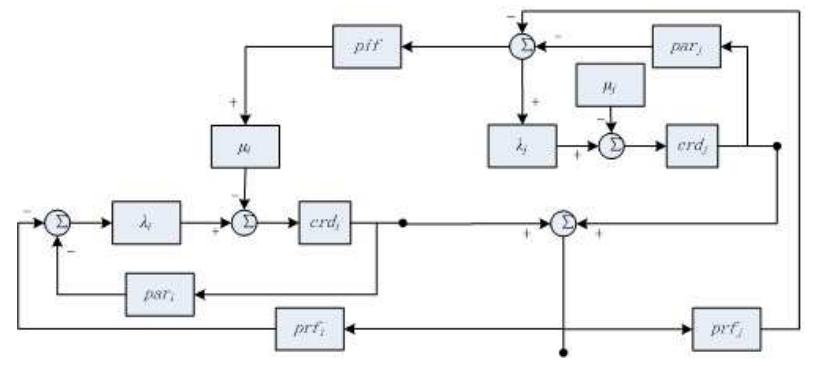

Fig. 5: The flow chart of noise effect in lumped controller with two units

From this expression, we can see that the correct factor of the $n+1$ control unit before $j\left(r_{j-n}^{t+n}\right)$ is related to the crowded degree of its downstream unit $\left(c r d_{j-n}\right)$, and there exists time delay. Q.E D

\subsection{The lumped controller}

In Multi-Control Agency, the control of each control unit to its arrival rate are all tend to realize their expected crowded degrees, which may affect the shunt rate of whole system. Actually, there is usually a total scheduler, which can coordinate the crowded degrees of units from system level. Thus, we monitor the system steady-state behavior based on the control agency, and constitute the lumped controller through modifying the crowded degree of each control unit.

Definition 4 The Lumped Law ccr: The crowded degree fluctuation from a single control unit to the average level of whole system. We use the correct number here to measure: $c c r=c r d_{i}-A V G\left(c r d_{i}\right)$.

Definition 5 System Imbalance sblc: The stability of crowded degree of control unit, we use standard deviation to measure:sblc $=\sqrt{\frac{1}{n-1} \sum_{i=1}^{n} c c r_{i}^{2}}, n$ is number of control units. System Imbalance reflects the stability of the lumped controller. And the smaller the System Imbalance is, the better the stability of system is.

Property 2 Noise Effect: Because of Domino Effect, the regulation of the crowded degree of any control unit will affect the stability of the lumped controller.

Proof: The original parameters in a lumped controller with two units are shown in Figure 5. Assume that it is balanced at original, that is $c r d_{i}=c r d_{j}=0$, sblc $=0$. Prove that the stability of system will be affected (that is, $s b l c \neq$ $0)$.

From the original condition, we can see that if $s b l c \neq$ 0 , it is only need to prove $\Delta c r d_{i} \neq \Delta c r d_{j}$. Given that the crowded degree of control unit $i$ is:

$$
\operatorname{crd}_{i}=\frac{1}{1+e^{-2\left(R_{i}-5\right)}}\left(R_{i}=\frac{x_{i}}{C_{i}} \mid x_{i}=\lambda_{i}-\mu_{i} \leq C_{i}\right)
$$

Since Domino Effect, the increment of control unit $i$ is $\Delta u_{i}=-p i f \cdot\left(c c r_{j}^{\prime}+\operatorname{par}_{j} \cdot \Delta c r d_{j}\right)$, then its crowded degree will be:

$$
\operatorname{crd}_{i}^{\prime}=\frac{1}{1+e^{-2\left(R_{i}^{\prime}-5\right)}}\left(R_{i}^{\prime}=\frac{x_{i}^{\prime}}{C_{i}} \mid x_{i}^{\prime}=\lambda_{i}-\mu_{i} \leq C_{i}\right)
$$

Assume that $\Delta c r d_{i} \neq \Delta c r d_{j}$ is true, then $\Delta c r d_{i}=\operatorname{crd}_{i}^{\prime}-\operatorname{crd}_{i} \neq \Delta c r d_{j}$, that is, $\operatorname{crd}_{i} \neq \operatorname{crd}_{i}+\Delta c r d_{j}$, substitute Eq.1 and Eq.2:

$$
\begin{gathered}
\frac{1}{1+e^{-2\left[\left(\lambda_{i}-u_{i}+\Delta u_{i}\right) / C_{i}-5\right]}} \neq \frac{1}{1+e^{-2\left(\lambda_{i}-u_{i}\right) / C_{i}-5}}+\Delta c r d_{j} \\
1+e^{-2\left[\left(\lambda_{i}-u_{i}\right) / C_{i}-5\right]} \neq 1+e^{-2\left[\left(\lambda_{i}-u_{i}+\Delta u_{i}\right) / C_{i}-5\right]}+ \\
\left(1+e^{-2\left[\left(\lambda_{i}-u_{i}\right) / C_{i}-5\right]}\right) \cdot\left(1+e^{-2\left[\left(\lambda_{i}-u_{i}+\Delta u_{i}\right) / C_{i}-5\right]} \cdot \Delta c r d_{j}\right)
\end{gathered}
$$

Because $e^{-2\left(\lambda_{i}-u_{i}\right) / C_{i}-5} \neq 0$, then divide both sides of the inequation above by $e^{-2\left(\lambda_{i}-u_{i}\right) / C_{i}-5}$, after reduct, we can get:

$$
\begin{gathered}
1 \neq e^{-2 \Delta \mu_{i} / C_{i}}+\left(1+e^{2\left[\left(\lambda_{i}-\mu_{i}\right) / C_{i}-5\right]}+e^{-2 \Delta \mu_{i} / C_{i}}+\right. \\
\left.e^{-2\left[\left(\lambda_{i}-\mu_{i}+\Delta \mu_{i}\right) / C_{i}-5\right]}\right) \cdot \Delta c r d_{j}
\end{gathered}
$$

If $\Delta c r d_{j}>0$, the crowded degree of unit $j$ will higher than the mean level, then $c c r_{j}^{\prime}>0$, that is, $\Delta u_{i}=-p i f\left(c c r_{j}^{\prime}+\operatorname{par}_{j} \cdot \Delta c r d_{j}\right)<0$, in Eq.3, $e^{-2 \Delta u_{i} / C i}>1$, $\left(1+e^{2\left[\left(\lambda_{i}-u_{i}\right) / C_{i}-5\right]}+e^{\left.-2\left[\left(\lambda_{i}-u_{i}\right)+\Delta u_{i}\right) / C_{i}-5\right]}\right) \cdot \Delta c r d_{j}>0$, the inequation is true. As above, if $\Delta c r d_{j}<0$, then $c c r_{j}^{\prime}<0, \Delta u_{i}>0, \quad$ in Eq.3, $e^{-2 \Delta u_{i} / C i}<1$, $\left(1+e^{2\left[\left(\lambda_{i}-u_{i}\right) / C_{i}-5\right]}+e^{\left.-2\left[\left(\lambda_{i}-u_{i}\right)+\Delta u_{i}\right) / C_{i}-5\right]}\right) \cdot \Delta c r d_{j}>0$, the inequation is true. Q.E D

\section{Example analysis of dynamic fuzzy feedback control system}

\subsection{Feasibility analysis of fuzzy control model}

Consider a scenic spot, assume its capacity is 100, and the expected crowded degree $c r d^{*}=0.6$. Set the adaptive law at instant $t_{i}:$ par $_{i}=44-t_{i-1}$, collect its shunt rate in a period of time, the initial value of arrival rate is assumed to be 20 .

We use MATLAB7.0 to simulate the expected arrival rate under a given shunt rate, and this is also the demand forecast. The curve of arrival rate and its shunt rate are shown in Figure 6, the part in black circle ([0-14] time) is called the system adapting period. Find out the crowded degree of this attraction under actual schedule, and compare it with the crowded degree under the best scheduling measure. The effect is shown in Figure 7.

From Figure 7, we can see that the crowded degree of attraction is fluctuating since the delay effect of real 


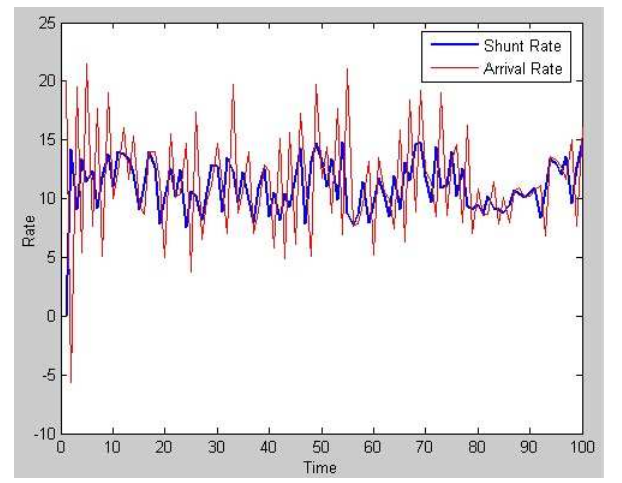

Fig. 6: Tracking effect of arrival rate to shunt rate

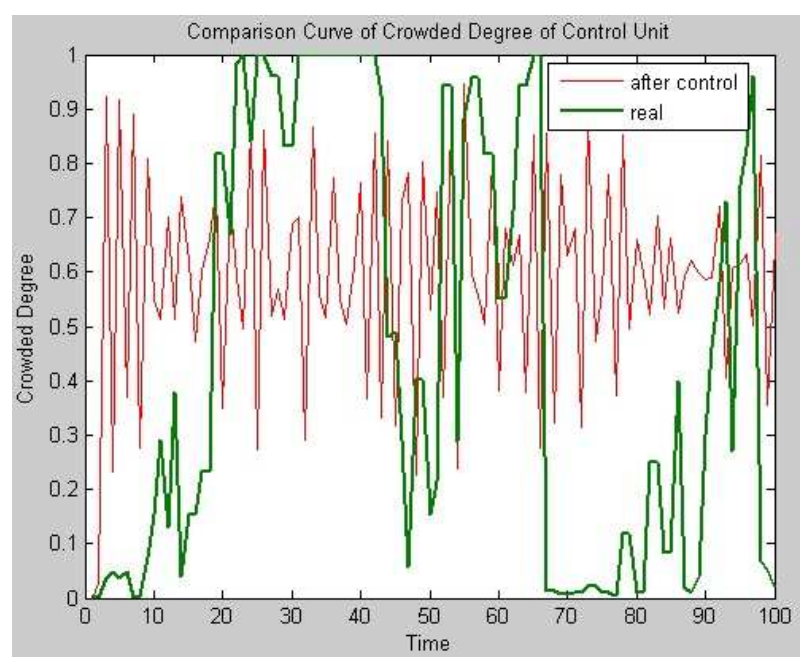

Fig. 7: Comparison of crowded degree of unit

schedule. The mean value of crowded degree is 0.47 , and the variance is 16.41 . The fuzzy feedback control can realize adaptive control, and its value is around the expected crowd degree with mean value 0.59 and variance 4.03. With the real-time feedback control, the mean value of system crowded degree is increased by $25.5 \%$, the variance reduction of $75.3 \%$. This means that the system can take in more tourists, and its of great significance for improving the efficiency of scenic area.

Figure 8 shows the absolute tracking variance of arrival rate to shunt rate. With delay, the first 14 minutes are system adapting period, and the mean tracking error is 6.99 , take up about $61.2 \%$ of the average shunt rate. After stable, the mean tracking error is 3.29 , take up $30 \%$ of the average shunt rate average. The forecasted arrival rate has tracking ability to shunt rate, and there exist time delay since it is reverse control. We should do some advance while making up the schedule solution according to the actual transportation time.

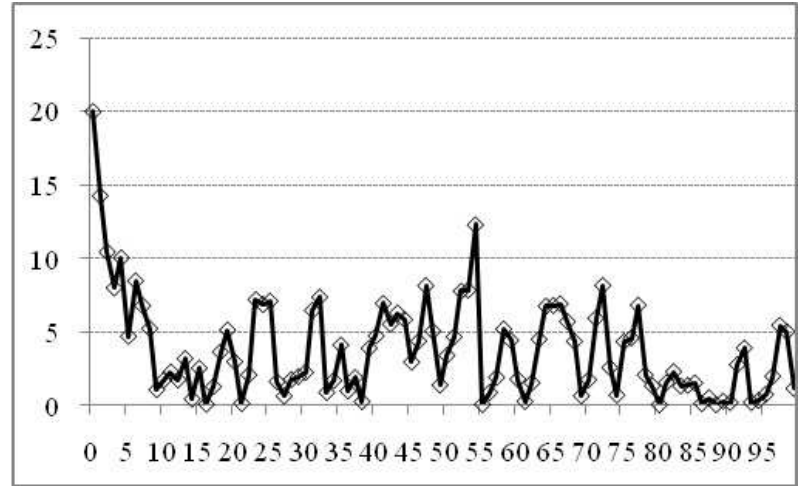

Fig. 8: Absolute tracking variance of arrival rate to shunt rate

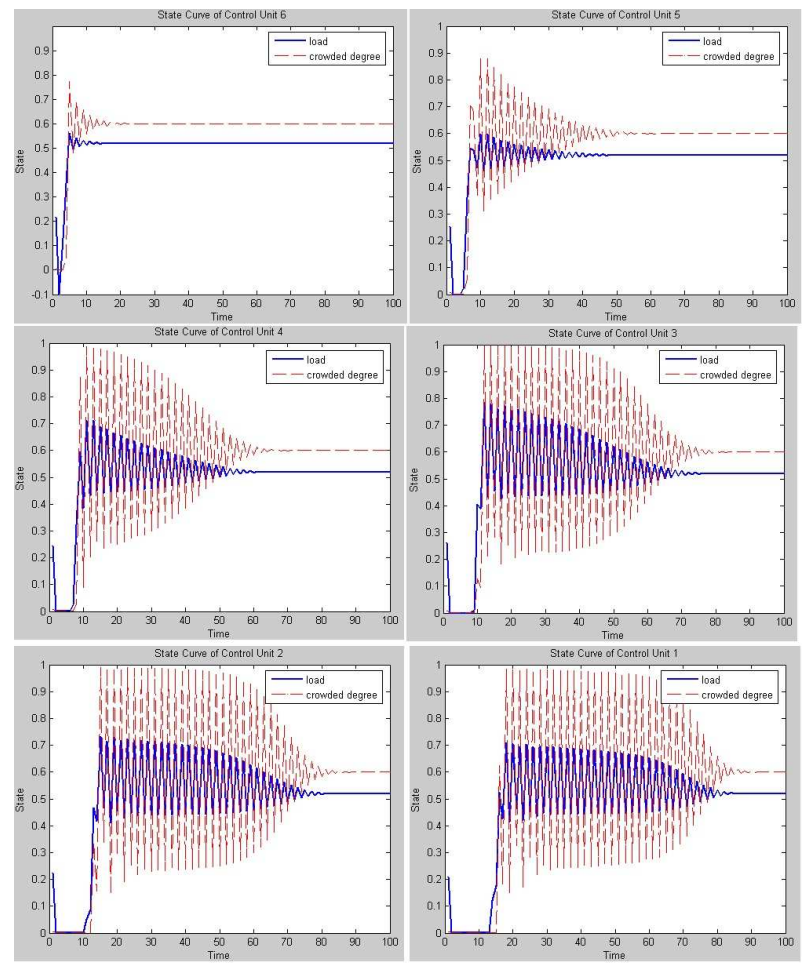

Fig. 9: The state performance of each unit in a fuzzy feedback control system with six units

\subsection{Performance test of dynamic fuzzy feedback control system}

Consider a fuzzy feedback control system with six units, set the capacity of control unit a random with interval [70100]. The state of each control unit is shown in Figure9. We can see that the states variations of the control units increase gradually with time delay due to the existence of the Domino Effect.

We compare the System Imbalance of six-control agency with the Lumped Controller in Figure10. It is 


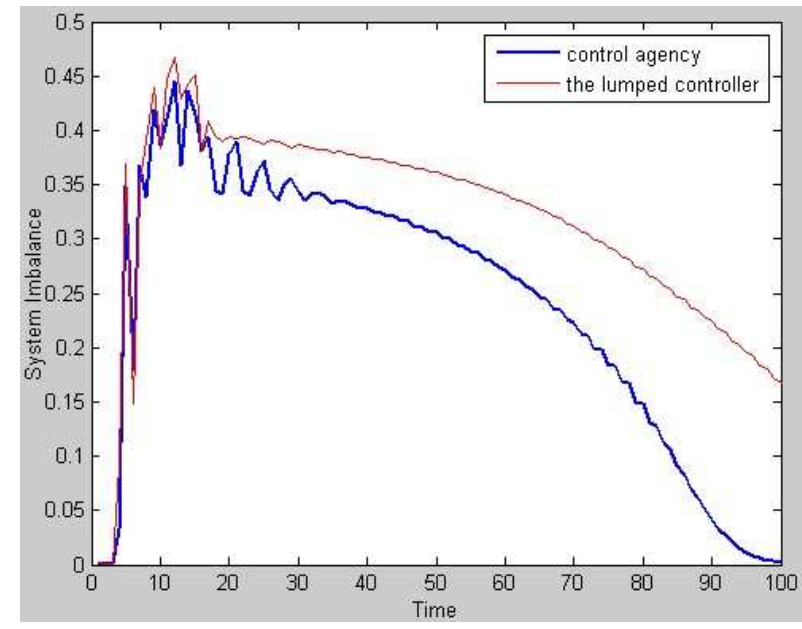

Fig. 10: Comparison of the system imbalance of six-control agency with the lumped controller

interesting that the balance rate of system slows down when we bring in the lumped law. We attribute it to the dynamic nature of the average crowded degree of system. Further research will consider setting the reference system according to the system scale (in this paper, we use real-time mean value of the system crowded degree), so as to speed up the convergence rate of the system.

Figure11 compares the system imbalances of lumped controllers with $\mathrm{n}$ control units $(n=2-9)$. We can see that in a given law, the system imbalance is mainly affected by the system dimension, and the more the system dimension is, the higher the system imbalance is (for example, the system imbalance of controller with eight units is $22 \%$ higher than controller with two units). We also found that the parity of the system dimension $n$ has a great influence on the variation of system imbalance (as seen in Figure11, the left is the system imbalance curve of lumped controllers with even units, and the right with odd units). The system imbalances of controllers with even units are smoother than the controller with odd units. We will do some further analysis for this phenomenon.

\section{Conclusion and prospect}

We use the fuzzy feedback control theory, bring in the adaptive law, external law, dumped law, construct the fuzzy control unit, multi-control agency and lumped controller according to the different dimensions. Presents and proves two kinds of effects: Domino effect and Noise effect. Finally, the simulation experiment on MATLAB7.0 show that the dynamic fuzzy predictive control model is well for tracking, and is more effective than the real schedule solution, the mean value of crowded degree is increased by $33.9 \%$, and the variance by $71.6 \%$. The performance test verifies two effects of the system.
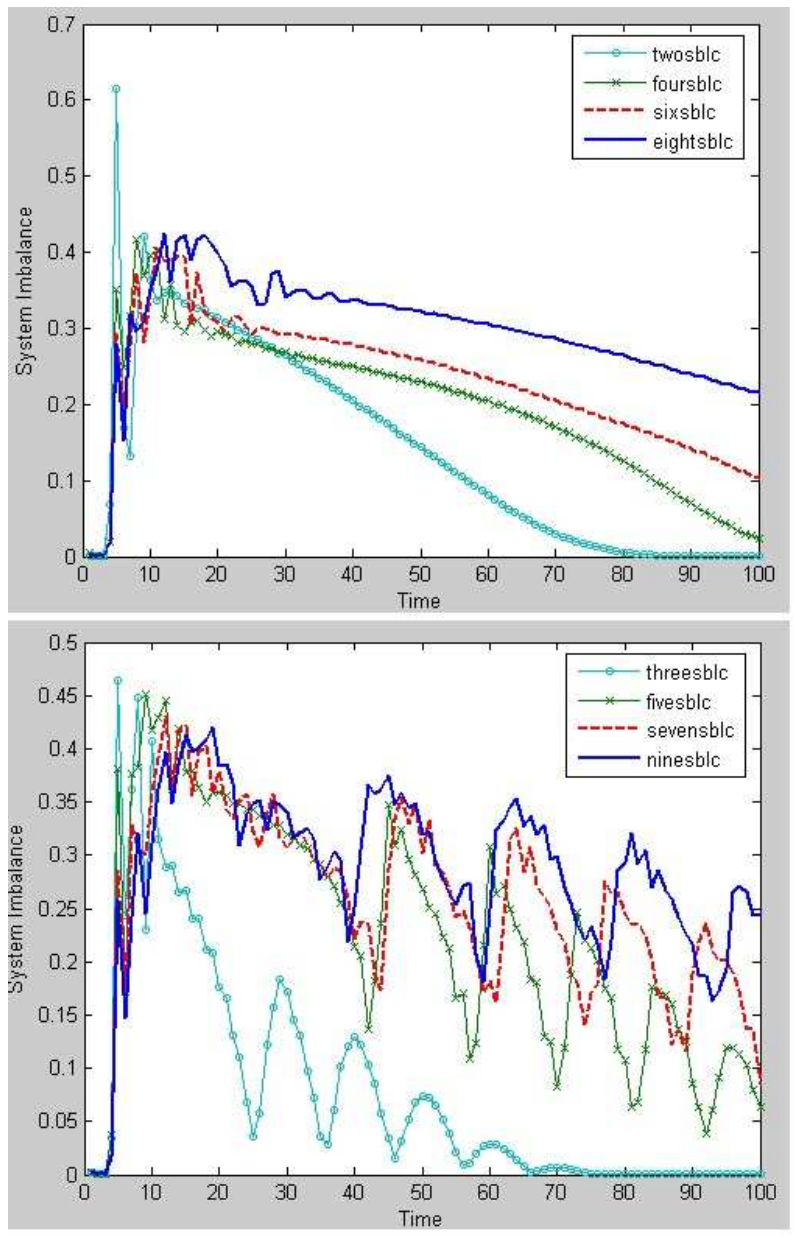

Fig. 11: Comparison of the system imbalance of the lumped controller with $n$ units

With time and length constraints, we will go in for issues below: 1. the scenic area diffluent system is a interactive network to its essence, we need to do more research on distributed fuzzy control in the future. 2. For tourists group, the time staying in a certain attraction is not only related to the individual differences, but also to the crowded degree of this attraction. The duration is an embedded random variable, and the function of duration and its effect on the shunt rate will be the second focus. 3 . The simulation show that the stability of the fuzzy control system performance is relevant to the parity of dimensions of the system, and this will be the third focus.

\section{Acknowledgement}

This work was supported by Major International Joint Research Program of the National Natural Science Foundation of China (71020107027), National High Technology Research and Development Major Program of China (Program 863) (2008AA04A107), the National 
Natural Science Foundation of China(71001075), Central University Fund of Sichuan Unversity No. skqy201112, and Doctoral Fund of Ministry of Education of China (20110181110034).

\section{References}

[1] Feng Gang, Ren Peiyu, Zhu Zhongfu, Ye Bin, Li Jianyu, Jian Daijun. Study on Comprehensive Performance Appraisal for Digital Jiuzhaigou Based on the Management Entropy, Tourism Tribune, 25, 72-78 (2010).

[2] Amy J. Liu, Guy N. Cameron. LANDSCAPE ECOL, 7, 581-595 (2001).

[3] Zhu Xiaohua, Wu En. Scientific Prospect of Fractal Theory Applied to the Network Space of Tourism System, Progress in Geography, 26, 133-142 (2007).

[4] Dongshan Wang, Guoguang He, China Civil Engineering Journal, 36, 68-74 (2003).

[5] Peng Qiyuan, Yin Yong, Yan Haifeng. Management Scheme of Sightseeing Vehicle on Tour Fastigium in Jiuzhaigou, Journal of Transportation Engineering and Information, 3, 1-5 (2005).

[6] Alexander M. Meystel, James S. Albus, Translated by Zuren Feng, Renhou Li et al. (Electronics Industrial Publishers, Beijing), (2005).

[7] Qian Jixin, Zhao Jun, Xu Zuhua. Predictive Control, (Chemical Industrial Press, Beijing), (2007).

[8] Chen S H, Yang Q, Wang C P, Impulsive control and synchronization of unifield chaotic system. Chaos, Solitons and and Fractals, 20, 751-758 (2004).

[9] J Zhu, Y P Tian. Stabilizing periodic solutions of nonlinear systems and applications in chaos control. IEEE Trans, On Circuits System, 52, 870-874 (2005).

[10] Yuping Tian, Jiandong Zhu, Guanrong Chen. A Survey on Delayed Feedback Control of Chaos. Journal of Control, (2005).

[11] Guoguang He, Yu Li, Shoufeng Ma. System Engineering Theory and Practice, 12 (2000).

[12] Mingbao Pang, Guoguang He. Simulation Study of Freeway Chaos System by Using Fuzzy Delay Feedback Control, (2009).

[13] T. Takagi, M. Sugeno. Fuzzy identification of systems and its applications to modeling and control. IEEE Trans Systems, (1985).

[14] Xiaoping Zhang, Zhongfu Zhu. An Analysis of Tourism Environment Capacity in Jiuzhaigou Scenic Area, Tourism Tribune, 22, 50-56( 2007).

[15] G. J. Kulawski, M. A. Brdys. Automatica, 36, 5-22 (2000).

[16] Jinkun Liu. Intelligent Control. (Publishing House of Electronics Industry, Beijing) 5 (2005).

[17] Gene F. Franklin, J. David Powell, Abbas Emami-Naeini. Translated by Qidan Zhu, Like Zhang, Xin Yuan etc. (Electronics Industrial Publishers, Beijing) 5 (2004).

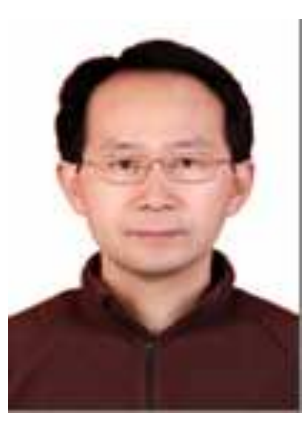

Peng

Ge

Ph.D., instructor, the deputy director of the institute of Sichuan University information and enterprise management, enterprise simulation lab director, with a multidisciplinary background including automation, computer, management science, industrial engineering, tourism management, and so on. Have strong organization ability, scientific research and abundant practice experience. Engaged in optimization scheduling, complex system modeling and simulation, the key theory and technology research on scenic area management in a long-term, have a long and in-depth tracking and theoretical research about the shunt peak scenic tourist management. Since 2009 , as the head of the corpus, mainly research the rate inquiry national high-tech development program (" 863 "plan) major projects (have finished) and the major international cooperation project of the National Natural Science Foundation of China.

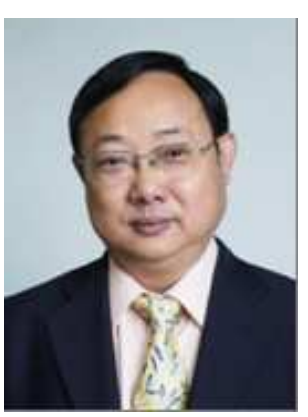

Peiyu Ren was born in Chongqing, China. He is a professor, Ph.D. supervisor. Prof. In recent years, he has presided over and completed five programs of NSFC, one project of State Social Science Found, one key project of Chinese Ministry of Education (Sub-Project), three ministerial and provincial projects and twenty-seven enterprises projects. At present, he is acting as the director of a large amount of projects and presiding over "Research on Low-carbon Scenic Integrated Management Model for Harmony Sustainable Development between Tourism Economy and Ecological Environment of Western Scenics" supported by Projects of International Cooperation and Exchanges NSFC(No.71020107027), "the RFID technology Based on Navigation Management Mode for Spatiotemporal Separation and Its Application in the Nature Conservation Region and Earthquake Relics Museum" supported by National High Technology Research and Development Program of China (No.2008AA04A107) and " Research on Sustainable Development Strategy and Evaluation System in Western Scenics " supported by Doctoral Fund of Ministry of Education of China(No. 20110181110034). In total, he has published 15 monographs and more than one hundred academic papers, including SCI, EI, Management World, China Industrial Economics and so on. 


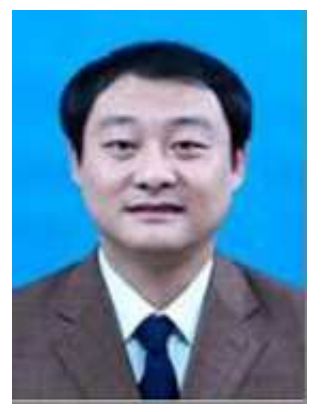

Maozhu

Jin

received his M.S. degree in the Department of Electronics Science and Technology and Ph.D. in the Department of Business from University of Huazhong Science and Technology, Wuhan, China, in 2005 and 2008, respectively. In April 2009, he joined Business School of Sichuan University, Chengdu, China. His research interests include multi-objective optimization, game theory, service science and supply chain management.

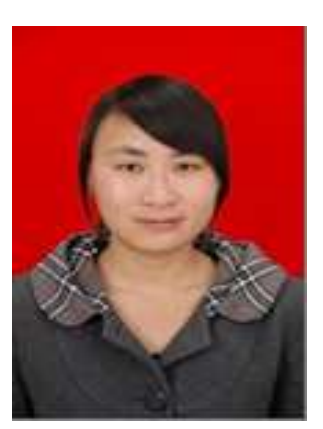

Yanqing Qiu is a doctoral student at Sichuan University majoring in Management Science and Engineering. Her researches mainly relate to System Simulation and Modeling, Logistics, Production Control, and Industrial Engineering, etc. In recent years, he has published about ten academic papers, some of which are retrieved by SCI, EI or CSSCI.

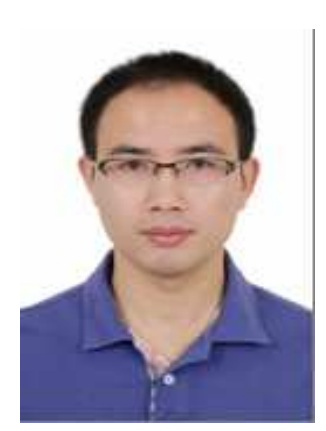

\section{Weimin Zheng} is a doctoral student at Sichuan University majoring in Management Science and Engineering, and is a member of Information and Business Management Institute of Sichuan University. His researches mainly relate to system simulation and modeling, system and information science and industrial engineering, etc. In recent years, he has published about ten academic papers, some of which are retrieved by SCI, EI or CSSCI. 